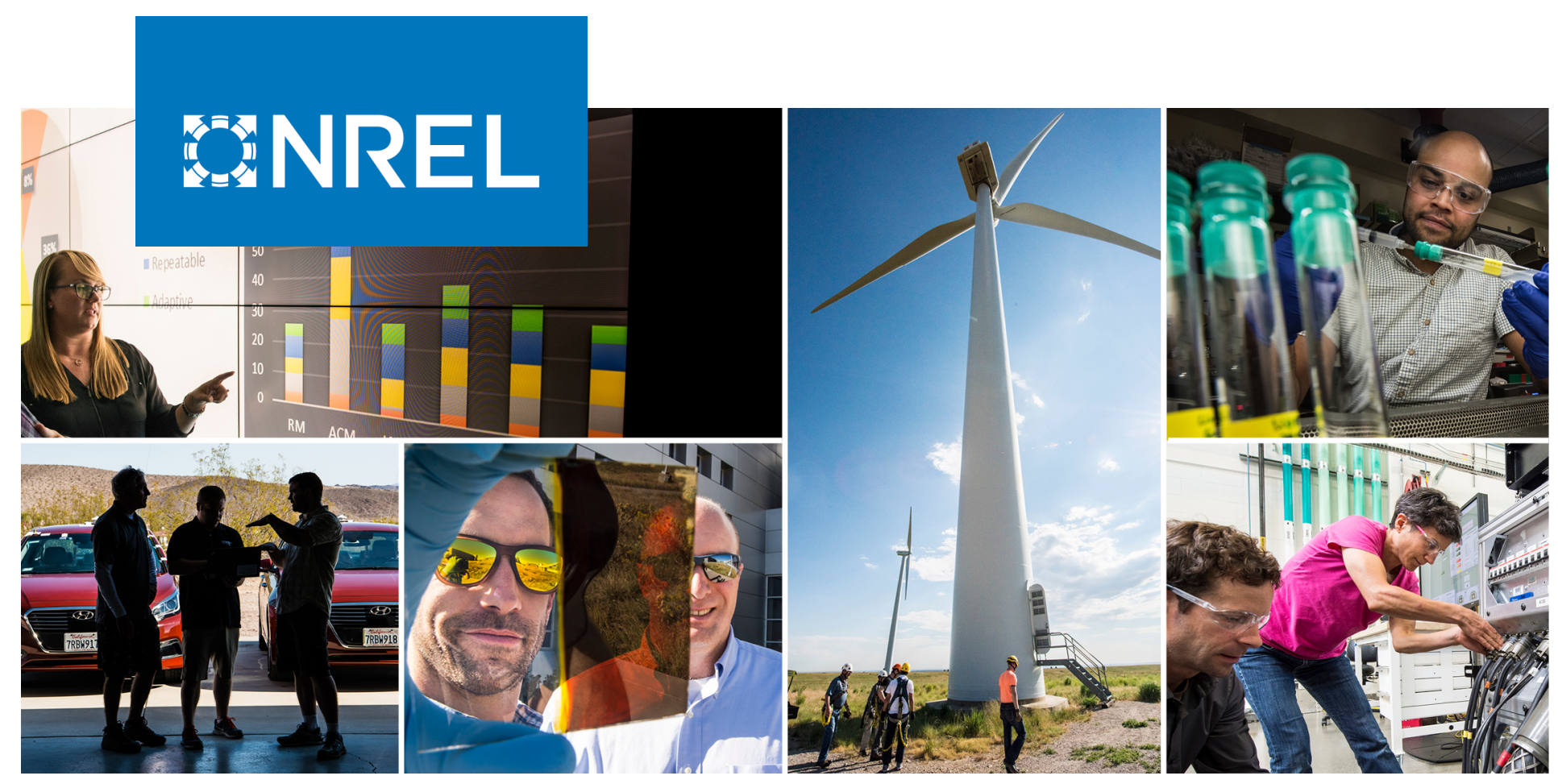

\title{
Developing a Reduced 240-Bus WECC Dynamic Model for Frequency Response Study of High Renewable Integration
}

\section{Preprint}

Haoyu Yuan, Reetam Sen Biswas, Jin Tan, and Yingchen Zhang

National Renewable Energy Laboratory

Presented at the 2020 IEEE Power and Energy Society Transmission and Distribution Conference and Exposition (IEEE PES T\&D)

October $12-15,2020$

NREL is a national laboratory of the U.S. Department of Energy Office of Energy Efficiency \& Renewable Energy

Operated by the Alliance for Sustainable Energy, LLC

This report is available at no cost from the National Renewable Energy Laboratory (NREL) at www.nrel.gov/publications.
Conference Paper

NREL/CP-5D00-74481

October 2020 


\title{
GNREL
}

\section{Developing a Reduced 240-Bus WECC} Dynamic Model for Frequency Response Study of High Renewable Integration

\section{Preprint}

\author{
Haoyu Yuan, Reetam Sen Biswas, Jin Tan, and \\ Yingchen Zhang
}

National Renewable Energy Laboratory

\author{
Suggested Citation \\ Yuan, Haoyu, Reetam Sen Biswas, Jin Tan, and Yingchen Zhang. 2020. Developing a \\ Reduced 240-Bus WECC Dynamic Model for Frequency Response Study of High \\ Renewable Integration: Preprint. Golden, CO: National Renewable Energy Laboratory. \\ NREL/CP-5D00-74481. https://www.nrel.gov/docs/fy21osti/74481.pdf.
}

(C) 2020 IEEE. Personal use of this material is permitted. Permission from IEEE must be obtained for all other uses, in any current or future media, including reprinting/republishing this material for advertising or promotional purposes, creating new collective works, for resale or redistribution to servers or lists, or reuse of any copyrighted component of this work in other works.

NREL is a national laboratory of the U.S. Department of Energy Office of Energy Efficiency \& Renewable Energy Operated by the Alliance for Sustainable Energy, LLC

This report is available at no cost from the National Renewable Energy Laboratory (NREL) at www.nrel.gov/publications.

Contract No. DE-AC36-08GO28308
Conference Paper

NREL/CP-5D00-74481

October 2020

National Renewable Energy Laboratory 15013 Denver West Parkway Golden, CO 80401

303-275-3000 • www.nrel.gov 


\section{NOTICE}

This work was authored by the National Renewable Energy Laboratory, operated by Alliance for Sustainable Energy, LLC, for the U.S. Department of Energy (DOE) under Contract No. DE-AC36-08GO28308. Funding provided by U.S. Department of Energy Office of Energy Efficiency and Renewable Energy Solar Energy Technologies Office. The views expressed herein do not necessarily represent the views of the DOE or the U.S. Government.

This report is available at no cost from the National Renewable Energy Laboratory (NREL) at www.nrel.gov/publications.

U.S. Department of Energy (DOE) reports produced after 1991 and a growing number of pre-1991 documents are available free via www.OSTI.gov.

Cover Photos by Dennis Schroeder: (clockwise, left to right) NREL 51934, NREL 45897, NREL 42160, NREL 45891, NREL 48097, NREL 46526.

NREL prints on paper that contains recycled content. 


\title{
Developing a Reduced 240-Bus WECC Dynamic Model for Frequency Response Study of High Renewable Integration
}

\author{
Haoyu Yuan, Reetam Sen Biswas ${ }^{1}$, Jin Tan, Yingchen Zhang \\ Power Systems Engineering center \\ National Renewable Energy Laboratory \\ Golden, CO, USA \\ \{haoyu.yuan, reetam.senbiswas, jin.tan, yingchen.zhang\}@nrel.gov
}

\begin{abstract}
The ongoing changes in the generation resource mix, driven by the rapid adoption of inverter-based resources (IBR) as well as the early retirement of synchronous generators, are bringing new challenges to the planning and operation of bulk electric power systems. Consequently, there is an increasing need to understand, design, and quantify the reliability service provision from IBRs by performing integrated scheduling and dynamic simulations. Test systems that have consistent scheduling and dynamic models rarely exist, however, largely because of the decoupled nature of the two simulations on a synchronous generator-dominated system. This paper develops the dynamic model of a reduced Western Electricity Coordinating Council (WECC) system. In conjunction with the existing scheduling model, it is suitable for integrating scheduling and dynamic simulations. The reduced 240-bus WECC model reflects the generation resource mix of the Western Interconnection as of 2018. Moreover, the developed dynamic model is validated against field frequency events measured by FNET/GridEye and preserves the dominant inter-area oscillation mode in WECC.
\end{abstract}

Keywords-dynamic simulation, generation resource mix, reduced WECC model, renewable energy.

\section{INTRODUCTION}

On March 16, 2019, utility-scale solar output in the California Independent System Operator region peaked at $10,765 \mathrm{MW}$ and was meeting 59\% of the total load [1]. And on May 24, 2013, Xcel Energy's Colorado system set a record of hourly wind energy penetration of $60.5 \%$ [2]. It is evidenced that the electric power system is dominated by inverter-based resources (IBRs) during light-load conditions in areas that aggressively adopt renewable energy resources (RES). The ongoing and fundamental changes in the generation resource mix bring new challenges to the planning and operation of the bulk electric power system.

The first challenge is that the electric grid needs for reliability services, such as inertia and governor response, are changing as RES penetration levels increase from one year to another and RES generation varies hourly throughout the day. The second challenge is that the technical and economic characteristics of RES determine that the provision and acquisition of reliability services will change drastically from current practices, which are tailored for synchronous generators. To properly address such challenges, integrated scheduling and dynamic simulations need to be performed to enable different stakeholders to understand and analyze both the economic and technical characteristics of RES.
However, publicly available, interconnection-level models suitable for integrated simulations are not present. Synthetic electric grid cases [3] developed through the Advanced Research Projects Agency-Energy grid data program include a 10,000-bus synthetic Western Electricity Coordinating Council (WECC) system; however, this model resembles only the footprint of the actual WECC system and misses necessary information, such as startup/shutdown costs, to perform a unit commitment study. The 179-bus reduced WECC system [4] was developed based on system conditions during the late 1990s and lacks scheduling information. The 240-bus reduced WECC system developed in [5] provides comprehensive scheduling information but lacks dynamic models. Moreover, the generation resource mixes in both systems do not reflect current grid conditions, which can be IBR-dominated.

The 240-bus WECC model in [5] is used as a starting point because of its comprehensive scheduling information, which is benchmarked against the operation data. On top of the model in [5], this paper first develops a 2018 base case that reflects the up-to-date generation resource mix [6]-[9], including installed photovoltaic (PV) and wind capacities; and then it develops high-fidelity dynamic models, including synchronous generators, exciters, governors, and renewable models that are validated against recorded events data of WECC. The National Renewable Energy Laboratory (NREL) will make the developed WECC 240-bus models publicly available to researchers and stakeholders. $^{2}$ Consequently, the developed base case and dynamic model, combined with the scheduling model in [5], serves as a good interconnection-level system to perform integrated scheduling and dynamic simulations and serves as a cornerstone to address the integration challenges of high penetrations of RES. The two main contributions of this paper are:

- $\quad$ Developed a 2018 WECC base case with the up-to-date resource mix that can simulate high renewable penetrations (up to $78 \%$ )

- Developed the dynamic model of the WECC system that captures the interconnection-level dynamic responses that are validated against recording data.

The remainder of this paper is organized as follows. Section II presents the generation resource mix using publicly available data. Section III introduces the development of the dynamic model. Section IV validates the dynamic model against field measurements. Section V concludes this work.

${ }^{1}$ Reetam Sen Biswas is also with Arizona State University.

\footnotetext{
${ }^{2}$ Link to the model will be available at https://github.com/harry-utk/240-BusWECC-Systems.
} 


\begin{tabular}{|c|c|c|c|c|c|c|c|c|c|c|c|}
\hline State & $\begin{array}{c}\text { Biomass } \\
(M W)\end{array}$ & $\begin{array}{l}\text { Coal } \\
(M W)\end{array}$ & $\begin{array}{c}\text { Geo- } \\
\text { thermal } \\
(M W)\end{array}$ & $\begin{array}{c}\text { Gas } \\
(M W)\end{array}$ & $\begin{array}{c}\text { Hydro } \\
(M W)\end{array}$ & $\begin{array}{c}\text { Nuclear } \\
(M W)\end{array}$ & $\begin{array}{c}\text { Pumped } \\
\text { Storage } \\
(M W)\end{array}$ & $\begin{array}{c}\text { Utility } \\
P V(M W)\end{array}$ & $\begin{array}{l}\text { Wind } \\
(M W)\end{array}$ & $\begin{array}{l}D P V \\
(M W)\end{array}$ & $\begin{array}{l}\text { State } \\
\text { Total } \\
(M W)\end{array}$ \\
\hline Arizona & & 6245 & & 16271 & 3527 & 4210 & & 2419 & & 1245 & 33917 \\
\hline California & 1008 & & 2788 & 44039 & 10061 & 2323 & 3746 & 15452 & 5690 & 7736 & 92843 \\
\hline Colorado & & 4948 & & 7829 & 672 & & 509 & 738 & 3106 & 349 & 18151 \\
\hline Idaho & 122 & & & 1272 & 2541 & & & 395 & 973 & & 5303 \\
\hline Montana & & 2488 & & 476 & 2671 & & & & 720 & & 6355 \\
\hline Nevada & & 809 & 751 & 8380 & 243 & & & 2423 & 152 & 284 & 13042 \\
\hline New Mexico & & 2817 & & 3750 & & & & 638 & 1682 & 139 & 9026 \\
\hline Oregon & & 642 & & 4366 & 4524 & & & 427 & 3213 & & 13172 \\
\hline Utah & & 4894 & & 3239 & 275 & & & 1407 & & 229 & 10044 \\
\hline Washington & 711 & 1460 & & 4162 & 25066 & 1200 & & & 3075 & & 35674 \\
\hline Wyoming & & 7254 & & 418 & 303 & & & & 1489 & & 9464 \\
\hline Alberta & & 13039 & & 9636 & 108 & & & 4410 & 541 & & 27734 \\
\hline B. C. & & & & 2650 & 10747 & & & & & & 13397 \\
\hline Mexico & & & 699 & 2140 & & & & & & & 2839 \\
\hline Grand Total & 1841 & 44596 & 4238 & 10826 & 60588 & 7733 & 4255 & 28309 & 21032 & 9982 & 290961 \\
\hline
\end{tabular}

\section{Generation Resource Mix BASEd on 2018 DatA}

The existing 240-bus system from [5] has a total capacity of $206 \mathrm{GW}$, including 2.0 GW PV and 6.6 GW wind. The IBR takes less than $5 \%$ of the total capacity. This resource mix is a representation of pre-2011 system conditions and is nowhere close to the current resource mix.

Therefore, resource mix and generation capacity in this 240bus system is updated according to publicly available data [6][9]. The updated resource mix for 11 U. S. western states, two Canadian provinces, and northern Baja Mexico is shown in Table I.

The 2018 Q3 data from the American Wind Energy Association [6] are referred for the wind capacity data. The utility-scale PV capacity is based on 2018 Q3 data from the Solar Energy Industries Association [7]. Small-scale PV (distributed PV; labeled DPV in Table I) capacity is based on 2018 Form EIA-861M from the U.S. Energy Information Administration [8]. Generation capacity from other fuel types (biomass, coal, geothermal, gas, hydro, nuclear, and pumped storage) are extracted from Form EIA-860 [9] where fuel type and capacity information of all generators in the United States are provided. The generation capacity of Alberta, British Columbia, and Mexico's northern Baja California Peninsula are based on a WECC planning case. Note that if the generation capacity of a fuel type of a state is less than $1 \%$ of that state's total capacity, the capacity of that fuel type is assumed to be 0 to prevent adding too many small generators. This explains some blank cells in Table I.

To match the generation capacity and mix shown in Table I, the generation capacity of the existing 240-bus system is scaled by a factor for each fuel and state combination. Some notable exceptions:

- The San Onofre nuclear plant in California was retired in 2013. Instead of scaling down the two nuclear generators in California, the San Onofre generator is removed in the new case.

- Instead of scaling down the hydropower plants in California, Helms PP, which is a pumped hydro storage plant in the field, is converted from hydro to pumped storage.
- Fifty-seven DPV generators (aggregated) are added in major load centers across the system.

- Three utility-scale PV plants are added in Lugo, Vincent, and Mira Loma. This decision is made based on the fact that there has been newly built PV generation near these stations since 2011 as a result of the Blue Cut fire and Canyon 2 fire events [10].

In the updated system, the total capacity is $291 \mathrm{GW}$, and the total IBR capacity is $59 \mathrm{GW}$, including utility PV, wind, and DPV, which comprise $20 \%$ of the total capacity. Consider that the peak demand of WECC is approximately $150 \mathrm{GW}$ [11] and light spring load is typically $50 \%-60 \%$ of the peak load, i.e., $75-$ $90 \mathrm{GW}$, so the highest IBR penetration of this test system can easily be reach $50 \%$ and become IBR-dominated. In theory, the highest penetration the developed system can simulate is $78 \%$, i.e., $59 \mathrm{GW} / 75 \mathrm{GW}$.

\section{THE DYNAMIC MODEL}

The dynamic model for the updated 240-bus system is developed in this section. The updated system has 146 generators with an average capacity of 1,800 MW, meaning that most generators are an aggregation of smaller generators of the same fuel type. With this level of aggregation, it is inevitable that some detailed or high-order dynamic responses will be lost; however, the dynamic responses at the system level should be preserved and validated.

To achieve this goal, first, generic dynamic models are determined based on fuel type. Then, to match the system frequency response, the inertia of the system is carefully distributed to match a WECC planning case. Finally, recorded measurements of real system events are used to validate the performance of the developed dynamic model.

All the dynamic models are chosen from the model library of Siemens PSS/E version 34.4 [12], and the simulations are performed on the same platform.

\section{A. Choices of Dynamic Models}

For each synchronous generator, a generator model, a turbine-governor model, and an excitation system model are modeled. Because of the aggregated nature of the generators, 
lower order dynamic models that are popular among researchers and open-source software are chosen.

The GENROU model, which is a round rotor generator model with quadratic saturation, is used for all synchronous generators. Default parameters from PSS/E [12] are used except for $\mathrm{H}$, the inertia time constants. The inertia constants have significant impacts on the system frequency response, which is a key concern in the IBR-dominant system. Therefore, this parameter is carefully chosen as detailed in Subsection III-B.

The SEXS model, which is a simplified excitation system, is used for all synchronous generators with the default parameters. Voltage response is not the focus of the integrated scheduling and dynamic simulations, and therefore a simplified model is chosen.

According to Table I, gas and hydro (including pumped storage hydro) generators comprise more than $50 \%$ of the capacity, and the rest of the synchronous generators (biomass, coal geothermal, and nuclear) can be classified as steam turbines. Therefore, to differentiate the frequency responses from these three types of generators, three turbine-governor models - GASTDU, HYGOVDU, and TGOV1DU - are used to represent gas, hydro, and other fuel type generators. Parameters of the governor models, such as droop settings and time constants, are important to system response and are tuned in Subsection III-C.

For nonsynchronous generators, state-of-the-art, secondgeneration generic renewable models are used [13], i.e., REGCA, REECB, and REPCA models. Such models include frequency response capabilities and are suitable for integrated scheduling and dynamic simulations. Note that advanced control capabilities are not enabled in the validating simulations because these IBRs are not currently providing such services.

\section{B. System Inertia}

For the reduced WECC system to have realistic inertial frequency response, the inertia constants of the generator models are carefully chosen so that the total kinetic energy of the reduced system matches that of a detailed WECC planning case. The 2015 high summer WECC planning case [14] is chosen for this purpose because the online capacity is close to the reduced WECC model. The net kinetic energy of the hydro and nonhydro generators of each area in the WECC planning case is mapped to that of the 240-bus test case. The contribution of the kinetic energy from hydro and non-hydro generation is summarized using the pie charts shown in Fig. 1 and Fig. 2.

Here, the objective is to find the inertia constants of the synchronous power plants in the 240-bus WECC system. Kinetic energy $(K)$ is the product of inertia constant $(H)$ and the installed capacity of a power plant $(S)$ [15]. Therefore, knowing the kinetic energy contributions from the hydro and the nonhydro generation in each area and the installed capacity of the generation in each area of the 240-bus system, the inertia constants of the hydro and non-hydro power plants of each area can be computed accordingly.

To observe the differences between the computed inertia constants and the average inertia constants of the synchronous units of the WECC planning case, we present a comparison

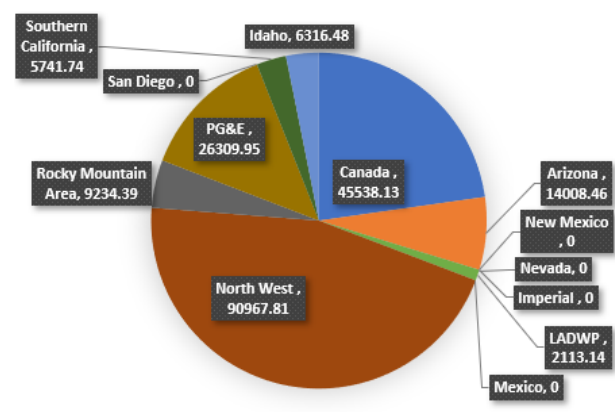

Fig. 1. Kinetic energy contribution from hydropower plants.

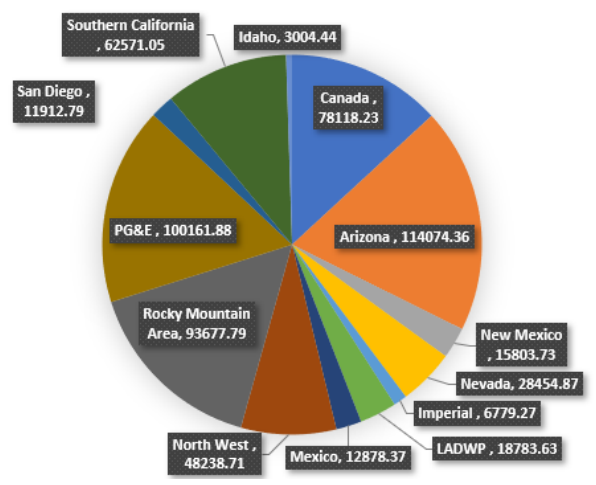

Fig. 2. Kinetic energy contribution from non-hydropower plants.

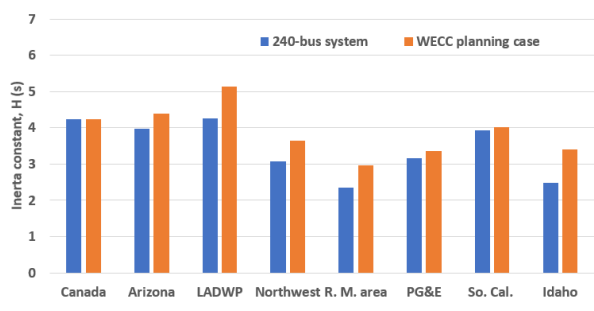

Fig. 3. Comparison of inertia constants in 240-bus system and WECC planning case for hydropower generation.

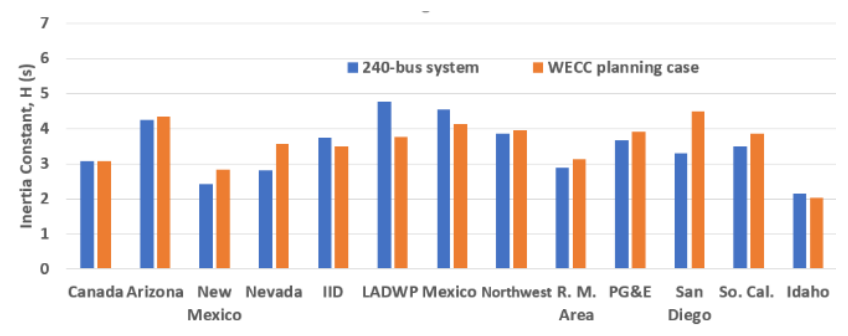

Fig. 4. Comparison of inertia constants in 240-bus system and WECC planning case for non-hydropower generation.

using the bar charts in Fig. 3 and Fig. 4. In the two figures, "R. M. Area" and "So. Cal." refer to the Rocky Mountain region and Southern California, respectively.

After the inertia of the 240-bus system is mapped, the inertial response (first 2 to 3 seconds after the disturbance) of the reduced system matches the actual system's response.

\section{Fine Tunings of Governor Models}

The governor models are fine-tuned to preserve the systemlevel frequency response, especially the settling frequency and frequency nadir. Recorded measurements of system events in 
WECC as shown in Table II are benchmarked. To match the recorded system response, the following parameters are tuned:

- Governor deadband: the deadband of $\pm 0.036 \mathrm{~Hz}$ is implemented on all governor models per [16].

- Droop settings: 5\% droop is set for HYGOVDU, and 6\% droop is set for both TGOV1DU and GASTDU, which are typical values in the field.

- Baseload generators: even though all synchronous generators are equipped with governors, a percentage of the generators, especially nuclear generators, are baseload and will not respond to frequency events.

- Time constants: time constants of the governor models are fine-tuned. Particularly, $\mathrm{T}_{1}$ and $\mathrm{T}_{2}$ of GASTDU, $\mathrm{T}_{\mathrm{g}}$ and $\mathrm{T}_{\mathrm{W}}$ of HYGOVDU, and $\mathrm{T}_{3}$ of TGOV1DU are tuned.

The tuning process can be summarized with the following four steps:

Step 1: The governor deadband and droop settings are first set to the typical values.

Step 2: The non-baseload capacity is tuned to match the key frequencies, i.e., frequency nadir and settling frequency. The non-baseload capacities of each fuel type are changed proportionally in a $10 \%$ step size to match the settling frequency. The $40 \%$ non-baseload capacity setting yields the best match in terms of the summed deviations from the two key frequencies.

Step 3: The composition of the non-baseload capacity is changed to refine the frequency response. A $40 \%$ non-baseload capacity yields a good match on the settling frequency but a higher frequency nadir. To maintain the settling frequency and reduce the frequency nadir, the non-baseload capacity of slower hydro generators is increased and that of the faster steam generators is decreased by a similar amount. Because the capacity of each generator is large and fixed, the non-baseload capacity of each fuel type can be changed only in discrete steps, and different combinations must be explored to ensure that the changed capacity of both fuel types are close enough. After tuning, $69 \%$ of hydro capacity together with $16 \%$ of steam capacity yields the best results.

Step 4: Finally, the time constants of the governor models are tuned to match the times when the frequency nadir and settling frequency are recorded.

\section{VALIDATION OF THE DYNAMIC MODEL}

In this section, the frequency response of the reduced model is validated against three recorded events in WECC. Moreover, the N-S oscillation mode of the reduced system is analyzed and validated.

\section{A. Validation Against Frequency Events}

To ensure the accuracy of the dynamic model, the dynamic frequency response of the reduced WECC model is compared with the FNET/GridEye frequency measurement data [17], [18]. Three different generation trip events that occurred in 2015 are picked up for the validation. The time, location, and estimated trip amount are shown in Table II. The frequency response from six Frequency Disturbance Recorder (FDR) locations are
TABLE II. EVENTS AND ESTIMATED AMOUNT

\begin{tabular}{|c|c|c|c|c|c|}
\hline \multirow[t]{2}{*}{ Event } & \multirow[t]{2}{*}{ Date } & \multirow{2}{*}{$\begin{array}{c}\text { Time } \\
\text { (UTC) }\end{array}$} & \multirow{2}{*}{$\begin{array}{c}\text { Estimated } \\
\text { Generation } \\
\text { Trip (MW) }\end{array}$} & \multicolumn{2}{|c|}{$\begin{array}{c}\text { Estimated } \\
\text { Location }\end{array}$} \\
\hline & & & & lat & long \\
\hline $\mathrm{A}$ & $03 / 03 / 2015$ & $22: 24: 50$ & 783.5 & 47.9119 & -114.550 \\
\hline $\mathrm{B}$ & $04 / 05 / 2015$ & $19: 57: 52$ & 805.0 & 34.8553 & -115.209 \\
\hline $\mathrm{C}$ & $04 / 26 / 2015$ & $17: 53: 23$ & 605.0 & 48.7557 & -119.861 \\
\hline
\end{tabular}

TABLE III. FDR LOCATION AND BUS NO. IN 240-BUS SYSTEM

\begin{tabular}{|c|c|c|c|}
\hline No. & FDR No. & Observation Point & Bus No. \\
\hline 1 & 732 & Sacramento, CA & 8005 \\
\hline 2 & 768 & San Diego, CA & 2201 \\
\hline 3 & 757 & Walkerville, MT & 6202 \\
\hline 4 & 996 & Portland, OR & 4101 \\
\hline 5 & 766 & Layton, UT & 6503 \\
\hline 6 & 779 & Maple Falls, WA & 4201 \\
\hline
\end{tabular}

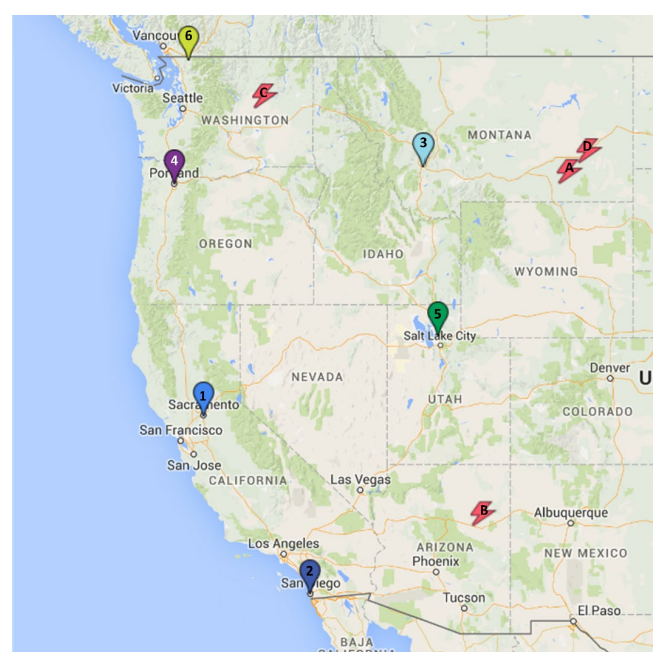

Fig. 5. Geographic distribution of events and obervation locations.

recorded, and six buses in the reduced model are compared with the FDR locations, as detailed in Table III. The locations of the events (lightning) and observations (circles) are plotted on a map in Fig. 5.

Under all three events, the simulation closely matches the measurement. Because of space constraints, only the comparison of Event A is depicted in Figure 6, which shows that the simulation (red curves) matches the measurement (blue curves) for all six locations. Specifically, the three key metrics to measure frequency response - inertial response (i.e., the rate of change of frequency), frequency nadir, and settling frequency - are compared. The reduced system can preserve the system-level frequency response, which is crucial for the integrated scheduling and dynamic simulations to address the challenge of the reduced inertia and governor response.

\section{B. Valiation of the $\mathrm{N}-\mathrm{S}$ Mode}

Inter-area oscillation modes in WECC need to be monitored and mitigated during planning and operation [19]. Siemens PSS SINCAL is used to perform an eigenvalue analysis of the reduced model. The well-known $\mathrm{N}-\mathrm{S}$ mode in WECC is observed in the reduced system (N-S mode A and B merge to one mode because of the lack of Alberta-to-U.S. connection [19]). The mode frequency is at $0.365 \mathrm{~Hz}$, and a $9.646 \%$ damping ratio is calculated. The mode shape is illustrated in Fig. 7, which shows that the generators in the north, e.g., Canada and Washington, are oscillating against the generators in the south, 

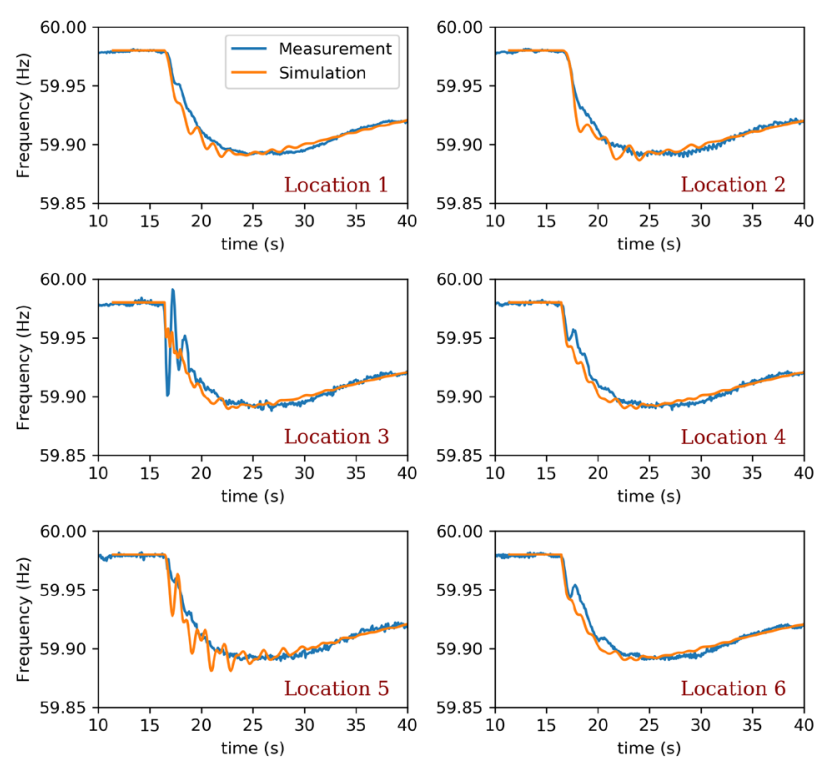

Fig. 6. Comparison of simulation and measurement for Event A.
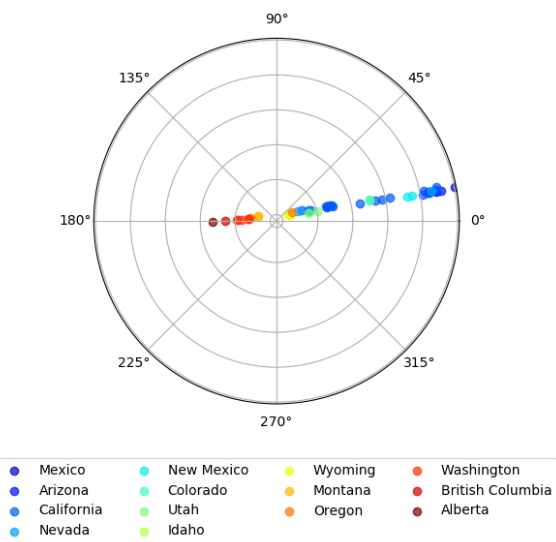

Fig. 7. Mode shape of N-S mode.

e.g., California. Based on the frequency and mode shape, it is validated that this mode represents the N-S mode [19].

Meanwhile, E-W mode and local oscillation modes around $1 \mathrm{~Hz}$ are calculated and have low damping ratios. Less damping oscillations can also be observed in the time-domain simulation shown in Fig. 6, which is expected because of the lack of stabilizer models. It is ongoing work to add stabilizers and to keep improving the small-signal stability of the system.

\section{CONCLUSION}

This work developed a reduced WECC model for integrated scheduling and dynamic simulation. This model updated the generation resource mix according to the 2018 state of WECC and therefore can simulate realistic IBR-dominated grid conditions with up to $78 \%$ penetration. Moreover, a dynamic model that preserves the system-level frequency response and the dominant N-S oscillation mode was developed, which is a first of its kind. This reduced WECC model will benefit both academia and industry audiences to address the challenges of integrating high penetrations of RES. Future work on the reduced WECC model will include developing power system stabilizer models, generating realistic time-series renewable and load data, and developing advanced inverter controls to enable grid services.

\section{ACKNOWLEDGMENT}

The authors gratefully acknowledge Dr. Yilu Liu from UTK for providing FNET data, Dr. Erik Ela from the Electric Power Research Institute, and Dr. Marcos Netto from NREL for their valuable discussion and help.

This work was authored by the National Renewable Energy Laboratory, operated by Alliance for Sustainable Energy, LLC, for the U.S. Department of Energy (DOE) under Contract No. DE-AC36-08GO28308. Funding provided by U.S. Department of Energy Office of Energy Efficiency and Renewable Energy Solar Energy Technologies Office. The views expressed in the article do not necessarily represent the views of the DOE or the U.S. Government. The U.S. Government retains and the publisher, by accepting the article for publication, acknowledges that the U.S. Government retains a nonexclusive, paid-up, irrevocable, worldwide license to publish or reproduce the published form of this work, or allow others to do so, for U.S. Government purposes.

\section{REFERENCES}

[1] S. Hanley, "California Solar Sets Record, But That's Not The Big News," Clean Tehnica, 24-Mar-2019.

[2] M. Goggin, "Xcel Colorado sets U.S. record with over $60 \%$ wind," Into the Wind the AWEA blog, 01-Nov-2013. .

[3] "Electric Grid Test Cases," Electric Grid Test Case Repository. [Online]. Available: https://electricgrids.engr.tamu.edu/electric-grid-test-cases/.

[4] J. Jung, Chen-Ching Liu, S. L. Tanimoto, and V. Vittal, "Adaptation in load shedding under vulnerable operating conditions," IEEE Trans. Power Syst., vol. 17, no. 4, pp. 1199-1205, Nov. 2002.

[5] J. E. Price and J. Goodin, "Reduced network modeling of WECC as a market design prototype," IEEE Power Energy Soc. Gen. Meet., pp. 1-6, 2011.

[6] "U.S. Wind Industry Third Quarter 2018 Market Report," American Wind Energy Association, Oct. 2018.

[7] "Solar Energy Industries Association (SEIA) for third quarter 2018." [Online]. Available: https://www.seia.org/states-map.

[8] "Small scale PV estimate, 2018 Form EIA-861M detailed data," U.S. Energy Information Administration.

[9] “Form EIA-860.” U.S. Energy Information Administration.

[10] "900 MW Fault Induced Solar Photovoltaic Resource Interruption Disturbance Report," NERC, 2017.

[11] "WECC State of the Interconnection," 2018. [Online]. Available: https://www.wecc.org/epubs/StateOfTheInterconnection.

[12] “PSSE 34.4 Model Library." Siemens PTI, Apr-2018.

[13] P. Pourbeik et al., "Generic Dynamic Models for Modeling Wind Power Plants and Other Renewable Technologies in Large-Scale Power System Studies," IEEE Trans. Energy Convers., vol. 32, no. 3, pp. 1108-1116, Sep. 2017.

[14] "WECC System Stability Planning Base Cases." [Online]. Available: https://www.wecc.org/SystemStabilityPlanning/Pages/BaseCases.aspx.

[15] A. Mullane, G. Bryans, and M. O'Malley, "Kinetic energy and frequency response comparison for renewable generation systems," in 2005 International Conference on Future Power Systems, 2005, pp. 1-6.

[16] "Industry Advisory, Generator Governor Frequency Response." North American Electric Reliability Corp., 05-Feb-2015.

[17] Y. Zhang et al., "Wide-area frequency monitoring network (FNET) architecture and applications," IEEE Trans. Smart Grid, vol. 1, no. 2, pp. $159-167,2010$.

[18] J. Tan, Y. Zhang, S. Veda, T. Elgindy, and Y. Liu, "Developing High PV Penetration Cases for Frequency Response Study of U.S. Western Interconnection," in 2017 Ninth Annual IEEE Green Technologies Conference (GreenTech), 2017, pp. 304-311.

[19] "Modes of Inter-Area Power Oscillations in the Western Interconnection," WECC Joint Synchronized Information Subcommittee, Jan. 2013. 